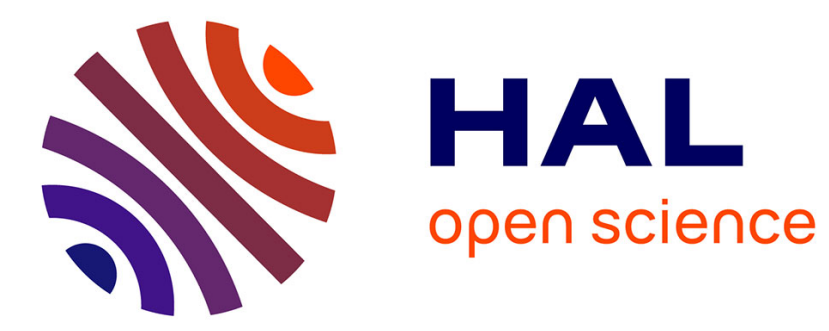

\title{
Le système fiscal français face aux réformes : essai de modélisation systémique de la fiscalité
}

Julien Defline

\section{To cite this version:}

Julien Defline. Le système fiscal français face aux réformes: essai de modélisation systémique de la fiscalité. Gestion et finances publiques : la revue - G\&FP - GFP, 2019, 5, pp.53-61. hal-03205634

\section{HAL Id: hal-03205634 \\ https://hal-amu.archives-ouvertes.fr/hal-03205634}

Submitted on 22 Apr 2021

HAL is a multi-disciplinary open access archive for the deposit and dissemination of scientific research documents, whether they are published or not. The documents may come from teaching and research institutions in France or abroad, or from public or private research centers.
L'archive ouverte pluridisciplinaire HAL, est destinée au dépôt et à la diffusion de documents scientifiques de niveau recherche, publiés ou non, émanant des établissements d'enseignement et de recherche français ou étrangers, des laboratoires publics ou privés. 


\title{
Le système fiscal français face aux réformes : essai de modélisation systémique de la fiscalité
}

\author{
Julien DEFLINE \\ Doctorant \\ Centre d'Études Fiscales et Financières (EA 891, GDR 1177 de Droit Comparé) \\ Aix-Marseille Université
}

«Il existe (...) une relation circulaire entre les trois

aspects de base des systèmes : les structures changent un instant lorsqu'elles fonctionnent, mais lorsque ce changement est si grand qu'il est nécessairement irréversible, un processus historique se développe, donnant naissance à une nouvelle structure. ${ }^{1}$

Les systèmes fiscaux sont définis par le Professeur Pierre BeLTRAME comme des « ensembles, plus ou moins cobérents, d'institutions, de règles et de pratiques fiscales (...) [faisant] incontestablement partie de notre univers social, économique et même psychologique $»^{2}$. Si les définitions du système fiscal français ne manquent pas, car elles sont présentes dans nombre d'ouvrages où les auteurs le conçoivent comme l'ensemble des impôts institués en France ${ }^{3}$, l'absence d'identification et d'analyse du système fiscal français d'après l'approche systémique rend ces définitions incomplètes.

L'étude du système fiscal français d'hier et d'aujourd'hui mène à un constat sans appel : la fiscalité actuelle est considérée comme désuète et complexe, en raison de l'inadaptation aux temps nouveaux malgré des modifications constantes. Les réformes ${ }^{4}$ successives n'ont pas permis de conserver un système efficient, moderne et adapté à la période contemporaine. Cette inefficience provient-elle d'échecs des gouvernants n'ayant pas conduit les réformes nécessaires à sa survie ou de facteurs exogènes à leur contenu ? Outre celle des constituants au XVIII ${ }^{\mathrm{e}}$ siècle et celle de l'impôt sur le revenu progressif de Joseph CAILlauX au début du $\mathrm{XX}^{\mathrm{e}}$ siècle, l'histoire postrévolutionnaire compta finalement peu de vraies «révolutions» fiscales ${ }^{5}$. Trois dates importantes ont marqué l'histoire de la fiscalité française au cours du XX ${ }^{\text {ème }}$ siècle : de 1914 à 1917

\footnotetext{
${ }^{1}$ J. A. MiLLER, «Basic concepts », The organizations in Behavioral science, 1972, vol. 17, n 1, janvier, p. 39, cité par J.L. Le Moigne, La théorie du système général, Théorisation de la modélisation, Paris, PUF, Système-Décisions, 1994, pp. 1820.

2 P. Beltrame, Les systèmes fiscaux, 3ème éd., Paris, PUF, Que sais-je ?, 1997, p. 3.

${ }^{3}$ L. Mehl et P. BeLtrame, Le système fiscal français, Paris, PUF, Que sais-je ?, 1995, p. 3.

${ }^{4}$ Cf. sur la notion de réforme: G. ORSONI, «De l'esprit de réforme et de quelques fondamentaux », in Réforme des Finances publiques et modernisation de l'Administration, Mélanges en l'bonneur de Robert HERTZOG, Paris, Economica, 2010, p. 408.

5 Sur l'histoire de la fiscalité, voir: N. Delalande et A. SPIRE, Histoire sociale de l'impôt, Paris, la Découverte, coll. Repères, 2010, 125 p. ; et N. Delalande, Les batailles de l'impôt : consentement et résistances de 1789 à nos jours, 2ème éd., Paris, Éd. du Seuil, L’Univers historique, 2014, 445 p.
} 
avec la création de l'impôt sur le revenu, 1954 avec la création de la taxe sur la valeur ajoutée, et 1990 avec la création de la contribution sociale généralisée. Mises à part celles-ci, l’histoire fut jalonnée de nombreuses modifications techniques, à finalité limitée, mais rares furent les réformes d'ampleur ces dernières années ${ }^{6}$. Récemment, le contenu des premières lois financières de la présidence MACRON se voulait majeur, telle une métamorphose de la fiscalité. Pourtant si les mesures sont nombreuses, la fiscalité française connait certes de grands changements, mais aucune « révolution ». Le Professeur Thierry LAMBERT reprenait déjà ce constat en 2002: «L'examen de notre système fiscal sur une longue période, la cinquième République, donne davantage le sentiment d'une continuité, indépendamment des alternances politiques, que de ruptures. Au nom des "contraintes" qui pèseraient sur le système fiscal, de "la difficulté à réformer" ou encore du "contexte international", la seule politique qui vaille, semble être celle de la continuité empreinte de résignation $»^{7}$. Ainsi, il convient de reprendre la conclusion de Georges DUMAS dans son ouvrage Politique fiscale : le naufrage: "Tous naviguent à vue, obnubilés par le court terme, sans cap et sans boussole: le naufrage $»^{8}$. La fiscalité française, tel un manuscrit maintes fois retouché par différents auteurs, mérite aujourd'hui une refonte globale. Il convient alors de s'interroger sur la réalisation de cette dernière.

Afin d'étudier l'influence des réformes fiscales sur la fiscalité, il convient d'approfondir d'une part, le but pour lequel ces changements et cette politique furent mis en œuvre, et, d'autre part, les raisons de l'absence de mise à plat de la fiscalité. À cette fin, une méthode particulière permettant de sortir d'un raisonnement subjectif et descriptif pour une réflexion plus normative est nécessaire pour « juridiciser $»^{9}$ la démarche et ne pas demeurer dans une étude descriptive des réformes. Le choix de l'approche systémique semble le plus adéquat pour dresser un panorama de la dynamique fiscale française. Il s'agit là d'une méthode interdisciplinaire élevant la réflexion à une analyse des relations entre des éléments, au-delà des éléments eux-mêmes. Pour ce faire, il convient

${ }^{6}$ Les réformes marquantes de la fiscalité de la seconde moitié du XXème siècle :

- 1948 : simplification des impôts et refonte de l'impôt sur le revenu (quotient familial, imposition par foyer, notion de domicile fiscal, etc.) et la création de l'impôt sur les sociétés ;

1959 : réforme de l'impôt sur le revenu et bases de la réforme de la fiscalité directe locale ;

1963 : mise en place de la fiscalité immobilière ;

1965 : réforme de la fiscalité des entreprises ;

1966 : élargissement de la TVA ;

1969 : réforme de la publicité foncière et des droits d'enregistrement ;

1973 : réforme de la fiscalité locale : taxe d'habitation et taxes foncières ;

1975 : création de la taxe professionnelle ;

1976 : institution de la taxation généralisée des plus-values ;

1979 : généralisation de la TVA;

1981 : institution de l'impôt sur les grandes fortunes.

7 Th. LAMBERT, «La politique fiscale sous la cinquième République : entre réformes et changements », in Th. LAMBERT et alii., Les chantiers fiscaux à engager, Paris, L'Harmattan, Finances publiques, 2002, p. 14.

8 G. Dumas, Politique fiscale : le naufrage, Paris, L'Harmattan, Finances publiques, 2007, p. 306.

9 Voir en ce sens : G. Teubner, Droit et réflexivité. L'auto-référence en droit et dans l'auto-organisation (trad. de l'allemand), Bruxelles, Bruylant et Paris, LGDJ, 1996, pp. 51-98. 
de leur appliquer la logique du système pour étudier la complexité de plusieurs objets. La systémique permet en effet de saisir le caractère complexe du droit et de le confronter à sa finalité. Deux intérêts propres à la méthode seront alors retenus. D'abord, elle permet de s'éloigner d'une approche essentiellement subjective revenant au simple commentaire des éléments, pour une méthode orientée vers davantage d'objectivité se concentrant sur les relations entre ces derniers. Le second intérêt réside dans le fait que cette méthode légitime une anticipation possible du résultat recherché. L'objectif de cette étude devenant une réflexion sur la finalité des réformes menées, cette démarche systémique demeure la plus appropriée. Michel TALY, dans son ouvrage Les coulisses de la politique fiscale ${ }^{10}$, relevait la nécessité de rechercher les finalités pour obtenir une réforme fiscale efficace et s'inscrivant dans un projet d'ensemble, pour en finir avec l'utilisation de la politique fiscale comme outil de communication. La fiscalité française mérite cohérence, simplicité et efficacité $^{11}$. Cette approche systémique, consentant à ne pas porter de jugement sur le fond mais sur la méthode, répond ainsi à la réflexion de Michel TALY.

La compréhension d’un système complexe passe nécessairement par sa modélisation ${ }^{12}$. Un système fiscal se concevant comme un système complexe, sa modélisation conduit à l'élaboration d'un modèle donné pouvant servir de référence. L'identification de modèles de systèmes reposant sur cette approche systémique parait la seule méthode capable d'appréhender toute leur complexité. Ces modèles respectent la complexité du réel et deviennent des facteurs efficients de résolution des problèmes organisationnels ${ }^{13}$. La modélisation représente un phénomène à l'aide d'un système.

L'application de la méthode systémique demande au préalable une définition du système telle que retenue dans cette étude. La notion de système se voit attribuer une grande disparité de significations ${ }^{14}$. Bien que beaucoup définissent un système comme un ensemble, un système n'est pas uniquement un ensemble ${ }^{15}$. "Qu'est-ce qu'un système? Et d'abord qu'est-ce qu'il n'est pas ?... Il ne s'agit pas d'un ensemble... Il est davantage qu'un système complexe $\rangle^{16}$. Si ce concept mal défini l'est de manière diverse et hétérogène, la doctrine semble pourtant s'accorder sur le fait qu'il s'agisse « d'un ensemble

\footnotetext{
${ }^{10}$ M. TALY, Les coulisses de la politique fiscale, Paris, PUF, 2016, 276 p.

${ }_{11}$ M. TALY, Les coulisses de la politique fiscale, Paris, PUF, 2016, p. 8.

12 D. Bourcier, «Sciences juridiques et complexité. Un nouveau modèle d'analyse », Droit et cultures, $\mathrm{n}^{\circ}$ 61, 2011, pp. 37-53.

${ }^{13}$ J.-L. Le Moigne, La modélisation des systèmes complexes, Paris, Dunod, 1990, 178 p.

${ }^{14}$ G. Timsit, «Système », in D. Alland et S. Rials (dir.), Dictionnaire de la culture juridique, Paris, PUF, Quadrige, 2003, p. 1462.

15 J.-L. LE Moigne, La théorie du système général, Théorisation de la modélisation, Paris, PUF, Système-Décisions, 1994 , pp. 18-20.

16 P. A. WeIss, L'archipel scientifique (trad. J. RAmBAUD), Paris, Maloine, 1974, p. 98.
} 
complexe constitué de parties liées entre elles par des relations stables $»^{17}$, soit de manière générale « un ensemble d'éléments en interaction $»^{18}$ ordonné et dynamique.

Étymologiquement, «système » vient du grec « Systema » : l'action d'arranger, de disposer en ordre. La définition d'un système retenue ici repose sur la coexistence de trois critères ${ }^{19}$ qui concomitamment réunis forment le système.

- Il faut tout d'abord un ensemble d'éléments ou de composants ${ }^{20}$. Les éléments du système peuvent être soit matériels soit abstraits, et classés en deux catégories : les données qualitatives et les données quantitatives ${ }^{21}$. Ces données quantitatives permettent d'exprimer la mesure des données qualitatives et ainsi de les rapporter à un référentiel particulier. Au sein d'un système, il existe différents types d'éléments, de niveaux différents qui peuvent avoir une organisation pyramidale ou circulaire. Certains sont des éléments de la structure du système, également qualifiés d'états repères, d'autres des sous-éléments composant ces éléments de la structure, eux-mêmes composés de sous-éléments, et ainsi de suite.

- Ensuite, ces éléments doivent entrer en interaction et représenter une certaine organisation dynamique au sein du système. L'interaction entre les éléments est un prérequis indispensable à l'existence d'un système puisque dans l'approche normative une juxtaposition d'éléments ne suffit pas. Ces interactions, qui forment une dynamique au sein du système, peuvent être diverses selon le type de système considéré. L'exercice de ces interactions doit permettre d'observer et de décrire la dynamique du système. Ce comportement dépendra des liens existants entre les éléments et surtout entre leurs caractéristiques fondamentales.

- Enfin, une certaine unité doit apparaittre entre ces éléments. Pour percevoir un système, les composants et leurs interactions doivent former un tout cohérent et logique. L'unité aide le système à conserver une certaine permanence au-delà des évolutions, et de lui attribuer une spécificité. Ce dernier critère permet également de rattacher un élément au système ou en dehors.

\footnotetext{
${ }^{17}$ G. Timsit, « Système », in D. Alland et S. Rials (dir.), Dictionnaire de la culture juridique, Paris, PUF, Quadrige, 2003, p. 1462.

18 P. DeLATTRE, Système, structure, fonction, évolution, Essai d'analyse épistémologique, Paris, Maloine-Doin, Recherches interdisciplinaires, 1971, p. 11.

${ }_{19}$ Ch. SAMPER, «Argumentaire pour l'application de la systémique au droit », Archives de philosophie du droit, $\mathrm{n}^{\circ}$ 43, 1999 , pp. 328-329. Mais aussi, P. ORIAnNe, Introduction an système juridique, Bruxelles, Bruylant, 1982, p. 24, ou encore P. Braillard, Théories des systèmes et relations internationales, Préface de P. FrIEDLANDER, Bruxelles, Bruylant, 1977 , p. 53. ${ }^{20}$ C. W. Churchmann, Qu'est-ce que l'analyse par les systèmes? ?, Trad. B. et M.-A. LeBLANC, Paris, Dunod, 1974, p. 43.

21 P. Delattre, Système, structure, fonction, évolution, Essai d'analyse épistémologique, Paris, Maloine-Doin, Recherches interdisciplinaires, 1971, p. 11.
} 
La coexistence de ces trois critères forme alors un système. Ainsi par assimilation, la combinaison de ces trois critères au sein de la fiscalité formerait un système fiscal.

Cette étude aura alors pour finalité l'essai de modélisation systémique de la fiscalité. Deux questions se posent auxquelles il conviendra de répondre: Quel système fiscal peut être identifié en France selon l'approche systémique? Et cette identification permet-elle de dresser une dynamique générale de la fiscalité au gré des réformes?

Pour répondre à ces interrogations, il sera nécessaire dans un premier temps d’identifier le système fiscal français comme modèle juridique, en s'éloignant de son sens ontologique auquel il a trop souvent été restreint, pour le caractériser dans son sens « déontologique » (I). L'idée d'une représentation systématisée étant interne au droit, la recherche d'un système fiscal le serait également et s'insérerait dans l'espace normatif. Dans un second temps, la recherche devra se concentrer sur la dynamique de ce système prédéfini, et sur l'influence des réformes menées par les gouvernants sur la fiscalité qui ont bien du mal à bouleverser le système; système qui sera qualifié de résilient (II).

\section{I - Une modélisation binaire}

Deux aspects peuvent être repris pour identifier le système fiscal. Le système fiscal ne peut être restreint aux seuls éléments qui le composent, il faut également y ajouter un jugement de valeur pour percevoir une dynamique en son sein. Si, bien des auteurs se contentent d'identifier de manière ontologique le système fiscal, donc uniquement par une description de ses éléments, cette définition n'en est que lacunaire (A). Le système étant un ensemble complexe, l’identification du système fiscal français mérite une approche plus large, « déontologique », pour l'appréhender de manière aboutie dans tous ses composants reflétant une dynamique $(\mathbf{B})$.

Ces deux sens proviennent de l'identification de la politique fiscale d'après Lucien MEHL dans la première édition de son ouvrage Science et technique fiscales ${ }^{22}$. Au sein de la doctrine, la politique fiscale connait deux sens qui, bien loin d'être opposés, se révèlent en fait complémentaires, et qui peuvent être transposés au système :

- Un sens descriptif d'abord, qui est qualifié d'ontologique. Ce sens purement descriptif comprend la politique fiscale comme l'intégralité des mesures prises se rapportant à un objet et à une période déterminée.

- À cela s'ajoute un sens normatif, que l'auteur qualifiait de « déontologique», porteur d'un jugement de valeur allant au-delà de la simple description des mesures. Le sens de

\footnotetext{
${ }^{22}$ L. MEHL, Science et technique fiscales, Paris, PUF, Thémis, 1959, p. 12.
} 
« déontologique » doit être compris comme un dérivé du terme « ontologique ». Il s'agit là d'ajouter le préfixe dé- au terme « ontologique » (dont son étymologie latine ontologia est composée de onto-, tiré du grec ancien $\omega_{v}$ (ôn, ontos) signifiant « étant, ce qui est », et de -logia, tiré du grec ancien $\lambda o ́ \gamma o \varsigma$ (logos) signifiant « discours, traité ») afin de signifier et d'exprimer l'éloignement, la négation, le contraire du sens ontologique ${ }^{23}$.

Ces deux sens reprennent l'ensemble des différents points de vue de la doctrine, puisque le premier était celui adopté par Louis TROTABAS et Jean-Marie COTTERET ${ }^{24}$, et le second par Maurice LAURÉ, dans son Traité de politique fiscale ${ }^{25}$. Cette modélisation binaire de la politique fiscale peut être transposée à la modélisation du système fiscal.

\section{A - Une identification ontologique lacunaire}

Le système fiscal français fait l'objet de peu de définitions précises appliquant la méthode systémique à la fiscalité dans une logique normative et non uniquement descriptive. En effet, la quasi-totalité des ouvrages portant sur le système fiscal français, notion générale et abstraite ${ }^{26}$, ne fait que décrire la fiscalité française dans tous ses éléments. La notion de «système fiscal» est couramment utilisée dans une définition lato sensu et non stricto sensu, assimilée en réalité à la fiscalité d'un État sans reprendre sa dynamique ${ }^{27}$. Cet usage, communément admis et repris depuis dans de nombreux manuels sur la fiscalité, conduit à une définition souffrant toutefois d'une certaine lacune lorsqu'il s'agit de modéliser la fiscalité de manière systémique. De cette façon, ils ne développent que les éléments, premier critère d'un système, et pensent établir le système fiscal français sans application de l'approche systémique. Ils ne font que le définir et l'identifier dans son sens ontologique c'est-à-dire comme l'intégralité des mesures fiscales d'un État. Les systèmes fiscaux sont «avant tout des systèmes nationaux dont les caractéristiques principales, le niveau et la structure des

\footnotetext{
${ }^{23} \mathrm{Il}$ ne s'agira pas ici d'utiliser le terme « déontologique » au sens de l'ensemble des règles ou des devoirs régissant la conduite à tenir pour les membres d'une profession ou pour les individus chargés d'une fonction dans la société, provenant de l'anglais deontology, issu en partie de deon en grec signifiant « ce qu'il faut faire ».

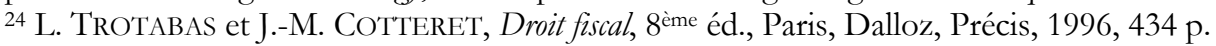

25 M. LAURÉ, Traité de politique fiscale, Paris, PUF, 1956, 425 p.

${ }^{26}$ M. Flamant, « Remarques sur l'évolution de la composition du prélèvement fiscal en France depuis cinquante ans », in J. Bouvier et J. WolfF (dir.), Deux siècles de fiscalité française XIX'e-XXe siècle, Paris, Mouton éditeur, Le savoir historique, 1973, p. 285.

${ }^{27}$ Voir en ce sens des études anciennes : J. THIRION, Étude sur la réforme du système fiscal, Montpellier, imprimerie De Cristin, Serre et Ricome, 1884, 16 p. ; R. STOURM, Systèmes généraux d’impôts, Paris, Librairie Guillaumin et Cie, 1893, 415 p. ; E. Brunet, Le système fiscal du grand-duché de Bade, Thèse pour le doctorat, Paris, Jouve, 1909, 175 p.; P. GrosBÉTY, Le nouveau système fiscal, Caen, imprimerie E. LANIER, 1918, 170 p. ; W.-C. YIN, Le système fiscal de la Chine, Paris, imprimerie du Montparnasse et de Persan-Beaumont, 1929, 212 p. ; R. THOUET, Vers la réforme du système monétaire et du système fiscal, Rouen, Maugard, 1938, 24 p. ; etc. Cette pratique étant reprise constamment depuis jusqu’à nos jours.
} 
prélèvements, reflètent les réalités historiques, politiques, sociales, économiques, institutionnelles et culturelles propres à chaque pays $»^{28}$.

Par cette vision du système, seul le premier des trois critères nécessaires à la constitution d'un système est repris : la fiscalité est bien composée d'un ensemble d'éléments ou de composants, que tout bon ouvrage détaille et qu'il est possible de regrouper en trois catégories d'éléments. Concernant les éléments de la fiscalité française, il s'agit des différents prélèvements établis en France que l'on peut qualifier et quantifier. Ainsi, les éléments du système fiscal français peuvent être regroupés en trois groupes d'éléments principaux :

- le groupe d'éléments : «imposition de l'enrichissement», regroupant comme éléments les impôts sur le revenu des personnes physiques et morales (impôt sur le revenu et impôt sur les sociétés), et les droits de mutation à titre gratuit (de succession et de donation) ;

- le groupe d'éléments : «imposition du patrimoine», regroupant comme éléments l'impôt sur la fortune immobilière, les taxes foncières et les taxes sur certains biens ;

- et le groupe d'éléments : «imposition sur la dépense », regroupant comme éléments la taxe sur la valeur ajoutée, les droits d'enregistrement des mutations à titre onéreux, les contributions indirectes, la taxe professionnelle et la taxe d'habitation.

Par cet usage courant du terme « système » en deçà de son sens réel et abstraction faite de toute dynamique au sein de ces éléments, l'étude des systèmes fiscaux souffre donc d'une grande lacune, car trop rarement poussée au-delà d'une simple description de la fiscalité étatique. Le système fiscal n'est pas qu'un ensemble d'éléments puisque ce qui fait de lui un système c'est davantage la dynamique qu'il existe entre ces éléments, que les éléments eux-mêmes. Pour combler cette identification lacunaire, il semble primordial d'aller plus loin et de définir le système fiscal français par son sens « déontologique ».

\section{B - Une identification « déontologique » aboutie}

Afin de définir le système fiscal français, il est nécessaire d'aller au-delà de son sens ontologique et d'étoffer la définition dans son sens «déontologique» pour appréhender son ensemble, et plus particulièrement la dynamique entre ces éléments. Il convient alors de rechercher si les trois critères définissant un système se retrouvent concomitamment réunis au sein de la fiscalité française.

28 A. VAlLÉE, Les systèmes fiscaux, Paris, Éditions du Seuil, Points, 2000, p. 7. 
Le critère de l'« unité » servira de postulat initial et sera supposé respecté. L’unité sera ici la même matière dans un même pays, c'est-à-dire la fiscalité française.

Le critère de l'« ensemble d'éléments ou de composants » est également rempli puisqu'il est apparu dès son sens ontologique et descriptif du système fiscal. Les éléments sont ceux décrits précédemment, regroupés en trois groupes d'éléments. Ces trois groupes d'éléments reflètent l'imposition de trois objets différents, tous reposants sur la notion de « richesses ». L'acquisition de richesses qui fait l'objet de l'imposition de l'enrichissement, la possession de richesses imposée dans le cadre de l'imposition du patrimoine, et enfin l'utilisation des richesses fiscalisée dans l'imposition de la dépense. Ces trois éléments principaux formant la structure du système fiscal français, suivent en réalité les éléments du système économique.

Le troisième critère enfin est celui de l'« interaction entre les éléments » afin de déterminer si la fiscalité française forme un système fiscal français. La détermination d'interactions entre ces éléments, étape trop souvent négligée, est essentielle. En effet, ces éléments doivent entrer en interaction pour parachever le système fiscal français. En cas d'absence d'interaction entre ces éléments, il n'existerait pas de système fiscal. En réalité, il n'en est rien puisque les trois groupes d'éléments principaux entrent en interaction et constituent les relations fiscales élémentaires. Il existe une interaction circulaire entre les trois groupes d'éléments structurels, qui reprend finalement le cycle économique, mais également des interactions pyramidales au sein de ces groupes d'éléments, entre les divers composants. Toute transformation d'un de ces groupes d'éléments, ou d'un élément de ces groupes d'éléments, aura des répercussions sur les autres éléments du système. Ainsi, toute modulation de l'acquisition des richesses entraîne inévitablement une évolution quant à la possession de ces richesses et in fine une métamorphose de l'utilisation de celles-ci. Il en va de même en cas de modification d'un élément des deux autres groupes d'éléments.

Ces relations au sein du système sont liées aux impositions pratiquées sur ces éléments. L'acquisition de richesses, qu'elle provienne du travail, de la cession de biens corporels ou incorporels, de la location de biens corporels et incorporels, du placement d'un capital financier, d'investissement dans une entreprise ou bien d'effet d'aubaine, sera imposée dans le cadre de l'imposition de l'enrichissement. L'imposition de l'enrichissement se fera soit par les droits de succession et de donation s'il s'agit d'une donation ou d'un héritage; soit par les impôts sur le revenu des personnes physiques et morales s'il s'agit de traitements, salaires, pensions, BIC, BNC, BA, revenus fonciers, revenus de capitaux mobiliers, plus-value ou bénéfices de société. Toute augmentation de l'imposition des richesses, qui se répercutera sur l'acquisition de ces richesses, aura pour conséquence une diminution du deuxième élément, à savoir la possession de richesses, et inversement. Il s'agit d'une première interaction entre des éléments socles du système fiscal. 
De la même manière, la possession des richesses - influencée par l'imposition de l'enrichissement (plus cette dernière sera faible, plus les contribuables posséderont des richesses) fait l'objet d'imposition dans le cadre de l'imposition du patrimoine, à travers l'impôt de solidarité sur la fortune (ISF) avant le $1^{\text {er }}$ janvier 2018 et de l'impôt sur la fortune immobilière (IFI) aujourd'hui, des taxes foncières et des taxes sur certains biens. L'augmentation de l'imposition sur le patrimoine diminuera l'utilisation des richesses, car chaque entité disposera de moins de richesses, et ainsi de suite. Il s'agit là de la deuxième interaction entre des éléments socles du système fiscal.

Enfin, l'utilisation de richesses - qui dépend de l'imposition de l'enrichissement et du patrimoine, car moins ces dernières seront élevées plus les richesses pourront être utilisées - qu'elle soit liée à la consommation ou à une mutation de propriété, se voit imposée dans le cadre de l'imposition sur la dépense (taxe sur la valeur ajoutée, droits d'enregistrement à titre onéreux, contributions indirectes, taxes professionnelles, et taxe d'habitation). Comme pour les deux autres interactions, toute transformation de l'imposition sur la dépense aura une incidence sur ce qui fait l'acquisition des richesses (travail, cession de biens corporels ou incorporels, location de biens corporels et incorporels, etc.). Il s'agit de la troisième interaction entre ces trois éléments qui referme ainsi le triangle, socle du système fiscal.

Les trois critères indispensables à l'existence d'un système fiscal français sont alors réunis. Dans un souci de clarification, l'identification du système fiscal français, telle que représentée dans cette étude, pourra être schématisée ainsi : 


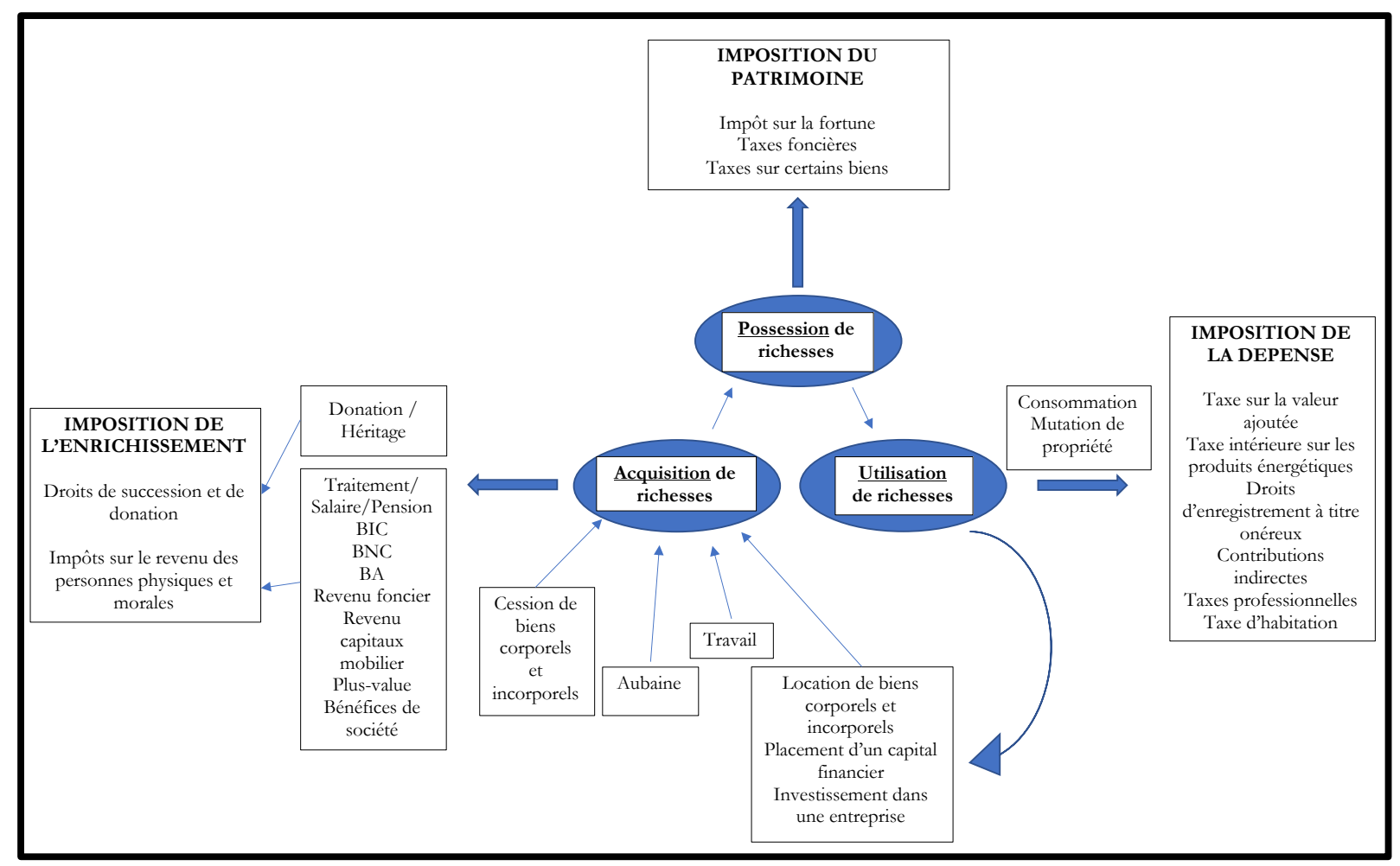

Système fiscal français ${ }^{29}$

Une fois le système fiscal français établi dans toutes ses acceptions, il convient de rechercher les raisons pour lesquelles les réformes fiscales ne parviennent pas à bouleverser notre système fiscal. Pour cela, l'étude de la dynamique du modèle «système fiscal » face aux réformes permettra d'en ressortir une caractéristique essentielle du système fiscal cause de cette inertie.

\section{II - Une modélisation résiliente}

L'étude de la dynamique du système fiscal français face aux différentes réformes fiscales mises en place par les gouvernants, passant par l'étude de l'influence de la politique fiscale sur les interactions, a établi que ce système était foncièrement résilient à deux égards. En premier lieu, le système fiscal français connaît une vraie résilience, car sa structure figée suit le cycle économique (A). En second lieu, l'inertie du système provient également en partie de cadres internationaux, cadres contraignant les gouvernants et limitant certains choix de politiques fiscales $(\mathbf{B})$.

\footnotetext{
${ }^{29}$ Le système fiscal français présenté ici reflète la pensée de l'auteur à l'issue de ses recherches. Nul doute que d'autres possibilités de représentation soient possibles et cohérentes, l'approche systémique permettant un vaste champ des possibles.
} 


\section{A - Une modélisation figée}

À travers les réformes et les politiques fiscales ${ }^{30}$ qu'ils souhaitent mettre en œuvre, les gouvernants espèrent engager de vrais changements au sein du système fiscal français. L'influence de ces nouvelles mesures sur le système reste à définir. Même si tout n'est pas refondu, de nombreux aspects du système changent grâce à ces réformes. Ces nouveautés tissent une nouvelle trame et modifient des composants du système fiscal actuel. Toutefois, elles ne suffisent visiblement pas pour changer de système et pour consacrer l'émergence d'un nouveau système. Un système, et notamment un système fiscal, se conçoit de manière plus profonde que par ses simples composants. Au-delà de nouveaux éléments issus des réformes, le système n'évolue que par les interactions élémentaires du système fiscal existant. En effet, la politique fiscale ne parvient pas à modifier ces interactions entre acquisition, possession et utilisation des richesses qui demeurent malgré les réformes, et donc entre l'imposition de l'enrichissement, l'imposition du patrimoine, et l'imposition de la dépense.

Afin d'éclairer ce constat, l'exemple des dernières lois de finances sera pris en illustration.

Le cas de la loi de finances pour $2018^{31}$ est une démonstration d'autant plus éloquente qu’à l'issue de la dernière élection présidentielle, les commentateurs constatent l'entrée dans ce qu'ils qualifient d'une «nouvelle ère », où l'exécutif prétend mener tous les changements dont le pays a besoin et notamment en matière fiscale. Les premières lois financières de la présidence MACRON tant attendues prévoient, il est vrai, de grands changements dans une vaste partie de la fiscalité française. Une nouvelle dynamique apparaittrait, une nouvelle logique, qu'il est intéressant de mettre en perspective avec celle de «l'Ancien Monde ». Les réformes fiscales du Président MACRON mises en place modifient l'équilibre du système à deux niveaux - à supposer que cet équilibre existe, ou à défaut un faisceau de tendances harmonieusement liées vers une situation d'équilibre jamais atteinte $^{32}$ - au niveau de l'imposition du patrimoine et de la dépense. En effet, les recettes de l'imposition de l'enrichissement restent globalement les mêmes malgré de nouvelles mesures (environ 13 milliards d'euros pour les droits de succession et de donation, 78 milliards d'euros pour l'impôt sur le revenu et 60 milliards d'euros pour l'impôt sur les sociétés en 2017 et 2018). Toutefois, en matière d'imposition du patrimoine, si les recettes des taxes foncières et des autres taxes restent relativement les mêmes entre 2017 et 2018, la suppression de l'ISF et la création de

\footnotetext{
30 «La politique fiscale recouvre l'ensemble des choix qui concourent à fixer les caractéristiques d'un système fiscal », in B. CASTAGNÈDE, La politique fiscale, Paris, PUF, Que sais-je ?, 2008, p. 3.

${ }^{31}$ Loi n $^{\circ}$ 2017-1837 du 30 décembre 2017 de finances pour 2018, JORF nº 0305 du 31 décembre 2017, texte $n^{\circ} 2$.

32 F. Trevoux, «La déformation du système fiscal par l'inflation », in J. Bouvier et J. WolfF (dir.), Deux siècles de fiscalité française XIX ${ }^{e}-X X^{e}$ siècle, Paris, Mouton éditeur, Le savoir historique, 1973, p. 308.
} 
l'IFI diminuent les recettes de l'imposition du patrimoine. Si en 2017 les recettes de l'ISF s'élevaient à environ 5,5 milliards d'euros, celles de l'IFI en 2018 n'étaient plus que de 1,25 milliard, soit une perte de 4,25 milliards qui augmente les richesses utilisables pour les contribuables. Il en va de même pour l'imposition de la dépense, alors que la majorité des recettes de ces prélèvements fiscaux restent stables, le dégrèvement progressif de la taxe d'habitation entraîne graduellement une baisse des recettes liées à cette imposition. En 2017, la taxe d'habitation rapporta environ 22 milliards d'euros. Le gouvernement prévoit d'ici 2020 de diviser progressivement par deux ces recettes (18 milliards d'euros en 2018, 15 milliards d'euros en 2019 et 11 milliards d'euros pour 2020), une diminution à long terme de 11 milliards d'euros pour l'imposition de la dépense. La taxe d'habitation devrait à terme totalement disparaître en 2023. L'exécutif espère qu'en libérant du pouvoir d'achat grâce à ces réformes, les capitaux afférents seront réinjectés dans l'économie, créant emplois et richesses. Le système fiscal français modifié n'en est pas pour autant nouveau.

L'exemple de la loi de finances pour $2019^{33}$ permet également de constater que malgré des réformes importantes le système fiscal conserve ses fondements. Alors que ces réformes font suite au mouvement des «Gilets jaunes »- mouvement sans précédent de contestation sociale dont les revendications étaient principalement d'ordre fiscal - elles modifient en partie le système fiscal mais ne le renversent pas. Ces réformes, s'inscrivant dans la poursuite des engagements de l'exécutif, reposent sur trois priorités : libérer l'économie et le travail, protéger les Français et investir pour l'avenir en préparant les défis de demain et en transformant l'action publique. Le texte, appuyé de la loi du 24 décembre $2018^{34}$ mettant en œuvre les mesures d'urgence économiques et sociales en réponse à la crise, compte plusieurs mesures fiscales importantes, pour une baisse des prélèvements obligatoires de 0,8 point afin d'atteindre un taux de 44,2\% du PIB cette année. Ces mesures répondant aux contestations d'injustice fiscale ne remettent cependant pas à plat la fiscalité et donc le système fiscal, car, si certains éléments en sont réformés, les groupes d'éléments du système et leurs relations semblent intacts.

Par ces mesures, la politique fiscale du Président MACRON modifie l'équilibre du système fiscal en transformant la répartition des recettes, mais le système conserve tout de même son essence et ses groupes d'éléments structurels. Les réformes fiscales viennent, par leurs caractéristiques, apporter des nouveautés fiscales qui, par voie de conséquences, affectent ce système façonné par des décennies d'histoire ${ }^{35}$. Si les modifications sont d'ampleur, le système

\footnotetext{
${ }^{33}$ Loi n $^{\circ}$ 2018-1317 du 28 décembre 2018 de finances pour 2019, JORF n ${ }^{\circ} 0302$ du 30 décembre 2018, texte ${ }^{\circ} 1$.

${ }^{34}$ Loi no $2018-1213$ du 24 décembre 2018 portant mesures d'urgence économiques et sociales, JORF n ${ }^{\circ} 0298$ du 26 décembre 2018 , texte $\mathrm{n}^{\circ} 1$.

${ }^{35}$ M. FLAMANT, « Remarques sur l'évolution de la composition du prélèvement fiscal en France depuis cinquante ans », in J. Bouvier et J. WolfF (dir.), Deux siècles de fiscalité francaise XIX $X^{e}-X X^{e}$ siècle, Paris, Mouton éditeur, Le savoir historique, 1973, pp. 284-307.
} 
fiscal, certes modifié et rééquilibré, conserve ses caractéristiques principales ainsi que la grande majorité de ses éléments. Finalement, que les réformes fiscales soient importantes, ou mineures, le système fiscal arrive à conserver ses fondamentaux étroitement liés in fine au cycle économique. Les trois groupes d'éléments précités demeurent, tout comme les interactions entre eux. Le système fiscal ne serait-il pas résilient, reprenant certaines logiques de l'autopoïèse ${ }^{36}$, s'étant construit luimême et maintenant son organisation malgré le changement des composants ? Le système fiscal, constituant l'armature de toute fiscalité ${ }^{37}$, détient une grande inertie qu'il est difficile de contrarier. Il survit malgré les changements, car, calqué sur le cycle économique, son ancrage extérieur le rend imperméable. L'unique remède résiderait dans la suppression de l’intégralité de la fiscalité française et dans la création d'une fiscalité totalement inédite, par exemple en instaurant un impôt unique comme développé par René STOURM ${ }^{38}$.

À la question de savoir si des réformes fiscales peuvent créer un nouveau système fiscal, la réponse sera négative sauf à supprimer l'intégralité des dispositions fiscales pour créer totalement un nouveau système fondé sur une nouvelle logique, ce qui n'a jamais été fait par les gouvernants sous la $V^{\text {ème }}$ République. Cette modélisation résiliente et le caractère inerte de sa structure fondé sur le cycle économique est la première cause de difficulté pour transformer le système fiscal français. Il convient alors de préciser la seconde cause contraignant le Gouvernement français dans ses choix de réforme fiscale : les cadres juridiques internationaux.

\section{B - Une modélisation influencée}

Le système fiscal français ne connait pas uniquement une inertie du fait de sa structure, il est également indirectement contraint et influencé par le cadre fixé par le droit de l'Union européenne ${ }^{39}$, ainsi que par certains standards fixés les organisations internationales.

\footnotetext{
${ }^{36}$ Voir sur l'autopoïèse et le droit : G. TEUBNER, Le droit, un système auto-poïétique (trad. de l'allemand), Paris, PUF, Les voies du droit, 1993, 296 p. ; ainsi que G. TEuBner, Droit et réflexivité. L'auto-référence en droit et dans l'auto-organisation (trad. de l'allemand), Bruxelles, Bruylant et Paris, LGDJ, 1996, 393 p. Le droit, donc également le droit fiscal, étant considéré comme un système autopoïétique par certains auteurs : «Le droit est même un système social autopoïtique de second degré. Il accède à ce statut en cristallisant une clôture opérationnelle indépendante à l'égard de la société, qui est un système autopoïétique de premier degré. Cette clôture opérationnelle s'instaure par la production auto-référentielle des composantes systémiques et par leur jonction dans un hypercycle » in G. TEUBNER, Le droit, un système auto-poütique (trad. de l'allemand), Paris, PUF, Les voies du droit, 1993 , p. 43.

${ }^{37}$ M. FlAmant, « Remarques sur l'évolution de la composition du prélèvement fiscal en France depuis cinquante ans », in J. Bouvier et J. WolfF (dir.), Deux siècles de fiscalité française XIX ${ }^{e}-X X^{e}$ siècle, Paris, Mouton éditeur, Le savoir historique, 1973, p. 285.

38 R. STOURM, Systèmes généraux d’impôts, 3ème éd., Paris, F. Alcan, 1912, 439 p.

${ }^{39}$ Voir en ce sens : P. MARCHESSOU et B. TRESCHER, Droit fiscal international et européen, Bruxelles, Bruylant, 2018,588 p.
} 
Le droit de l’Union européenne, facteur exogène à la fiscalité interne, empêche les autorités publiques d'intervenir dans certains domaines et les limite dans l'élaboration de leurs réformes fiscales. Le droit de l'Union européenne encadre les gouvernants dans leurs réformes et restreint ainsi les changements possibles de système fiscal, car ils se doivent de respecter à la fois les interdictions fiscales européennes, les règles d'harmonisation des législations fiscales établies, mais également les libertés fondamentales et les principes généraux du droit communautaire.

Toute réforme fiscale française se doit de respecter d'abord l'encadrement européen sur les interdictions fiscales ${ }^{40}$. La première d'entre elles est l'interdiction d'établir des droits de douane ou des taxes d'effet équivalent à des droits de douane ${ }^{41}$ afin de garantir la libre circulation des marchandises. La deuxième est l'interdiction de mettre en place des aides publiques ${ }^{42}$, qui impliquerait une distorsion de concurrence entre différents agents économiques. La dernière enfin est l'interdiction des impositions intérieures discriminatoires ${ }^{43}$ qui, comme l'interdiction précédente, vise à garantir des règles de concurrence saines nécessaires au fonctionnement du marché intérieur. Ainsi, toute modification du système fiscal français conduisant à établir une de ces interdictions est proscrite et limite alors les transformations possibles du système fiscal.

De même, les réformes fiscales ne peuvent pas venir modifier les règles internes harmonisées au niveau de l'Union européenne ${ }^{44}$. Cette harmonisation est assurée par le biais de directives communautaires qui empêchent les États de s'en écarter. L'Europe fiscale existe en matière d'impositions indirectes, la majorité des dispositions nationales sont en effet communes à tous les États membres en matière de TVA, mais également de droits d'accises. Toutefois, l'harmonisation des impositions directes peine à avancer. Elle stagne par l'absence d'unanimité des États qui ne parviennent pas à un rapprochement des législations et préfèrent conserver toute leur souveraineté en la matière ${ }^{45}$, la fiscalité étant souvent considérée comme la marque fondamentale de la souverainetét ${ }^{46}$. L'harmonisation des impositions directes reste alors faible et concerne

\footnotetext{
${ }^{40}$ Il s'agit ici de la problématique de l'intégration négative et positive des droits fiscaux nationaux développée par A. Maitrot de la MotTe dans sa thèse : A. Maitrot De LA MotTE, Souveraineté et construction communautaire : Recherche sur les impôts directs, Thèse pour le doctorat, Paris, LGDJ, Bibliothèque de science financière Tome 44, 2005, 537 p. Voir également: O. AllouARD, Les frontières fiscales dans l'Union européenne appliquées à la fiscalité des entreprises, Thèse pour le doctorat, Paris, L'Harmattan, Finances publiques, 2016, 558 p.

41 Articles 28 et 30 du Traité sur le fonctionnement de l'Union européenne (TFUE).

${ }^{42}$ Articles 107 à 109 du Traité sur le fonctionnement de l'Union européenne (TFUE). Voir en ce sens : I. PAPADAMAKI, Les aides d'État de nature fiscale en droit de l'Union européenne, Bruxelles, Bruylant, 2018, 539 p.

43 Article 110 du Traité sur le fonctionnement de l'Union européenne (TFUE).

${ }^{44}$ Voir en ce sens : D. VILLEMOT, L’harmonisation fiscale européenne, 2ème éd., Paris, PUF, «Que sais-je ?», 1995, p. 127 ; L. VALLÉE, «Heurts et malheurs de l'harmonisation fiscale européenne », CJEG, 2006, n 631, p. 201 ; Th. LAMBERT, "De l'harmonisation à l'intégration des législations fiscales nationales dans le cadre de l'Union européenne ", RTD Com, 2009, n³, pp. 517-528.

45 A. Maitrot De LA MotTe, Souveraineté et construction communautaire: Recherche sur les impôts directs, Thèse pour le doctorat, Paris, LGDJ, Bibliothèque de science financière Tome 44, 2005, 537 p.

${ }^{46} \mathrm{~J}$. BuISSON, «Impôt et souveraineté », Archives de philosophie du droit, 2002, n 46, pp. 25-31.
} 
uniquement certains aspects de la fiscalité des groupes de sociétés. Ainsi, l'harmonisation des impositions indirectes empêche les pouvoirs publics de modifier des éléments faisant partie du groupe de l'imposition de la dépense (TVA et droits d'accises). Quant à celle des impositions directes, les gouvernants ne seront pas totalement libres dans la réforme de certains éléments du groupe de l'imposition de l'enrichissement, notamment pour certains régimes fiscaux des groupes de sociétés.

Enfin, la politique fiscale spécifique à chaque État membre doit respecter la protection des libertés communautaires fondamentales (libre circulation des marchandises ${ }^{47}$, libre circulation des personnes $^{48}$, liberté d'établissement ${ }^{49}$, liberté de prestations des services ${ }^{50}$, et libre circulation des capitaux $^{51}$ ), ainsi que les principes généraux communautaires ${ }^{52}$. Le système fiscal ne saurait être transformé sans respecter ces mesures prévues par le droit de l’Union européenne.

Si le droit de l'Union européenne n'empêche pas toute modification de la fiscalité interne française, il vient restreindre le champ des possibles alors que le droit fiscal est l'un des pouvoirs régaliens de l'État par excellence. Le système fiscal ne peut totalement être remis à plat, car les politiques fiscales se devant de respecter les règles européennes, les gouvernants français ne peuvent modifier certaines dispositions (par exemple en matière de TVA) ni introduire de nouvelles mesures fiscales allant à l'encontre des interdictions européennes.

Outre ces cadres européens, les organisations internationales développent des standards que leurs États membres sont invités à respecter et notamment des standards fiscaux. Si ces normes ne sont en général juridiquement pas contraignantes pour les États, elles le sont économiquement en pratique, car les États ne les respectant pas se risquent à certaines représailles.

Ces organisations intervenant dans la création de normes fiscales sont principalement: l'Organisation de coopération et de développement économiques (OCDE), le Fonds monétaire international (FMI), la Banque mondiale et l'Organisation mondiale du commerce (OMC). Outre leurs missions de surveillance et de contrôle pour garantir un marché financier et économique meilleur, leur mission principale réside dans l'élaboration des normes internationales influençant le système fiscal des États. Ces normes peuvent être des «codes de bonne conduite » à l'instar du « code de conduite en matière de transparence des finances publiques » publié par le FMI ou des « lignes directrices pour la transparence budgétaire » publiées par l’OCDE. Il peut également s'agir

\footnotetext{
47 Article 34 du Traité sur le fonctionnement de l’Union européenne.

48 Article 45 du Traité sur le fonctionnement de l'Union européenne.

49 Article 49 du Traité sur le fonctionnement de l'Union européenne.

50 Article 56 du Traité sur le fonctionnement de l'Union européenne.

51 Article 63 du Traité sur le fonctionnement de l’Union européenne.

52 D. SimON, «Y a-t-il des principes généraux du droit communautaire », Droits, 1991, n 14, p. 73.
} 
de modèles de conventions fiscales internationales qui conduisent les États à souscrire à l'un de ces modèles $^{53}$. L'OCDE est l'un des acteurs majeurs de l'élaboration de normes fiscales internationales, dont le but principal est d'améliorer la coopération dans la lutte contre de la fraude et l'évasion fiscale pour éviter la concurrence fiscale dommageable entre États ${ }^{54}$. Pour cela, elle élabore un modèle de convention fiscale afin de régler les cas de double imposition et de non-imposition en cas d'élément d'extranéité. Une fois signées par la France, ces différentes dispositions seront contraignantes pour l'État qui ne saurait y déroger sauf à rompre ses engagements contractuels ${ }^{55}$. Le système fiscal français est alors en partie calqué sur ce modèle de convention, notamment pour les dispositions de fiscalité internationale créant en parallèle un système fiscal international. Façonné par ce modèle, le système en est d'autant plus résilient. Il peut enfin s'agir de normes, autres que les conventions, mais luttant également contre la fraude fiscale, contre les territoires non coopératifs, contre la concurrence fiscale dommageable, et prônant assistance et coopération entre les administrations des États ${ }^{56}$. Il en est par exemple ainsi du récent plan BEPS (Base erosion and profit shifting) de l'OCDE. Par ce plan, l'organisation tente de lutter contre les pratiques d'érosion de la base d'imposition et de transfert de bénéfices en équipant les gouvernements d'instruments nationaux et internationaux pour lutter contre l'évasion fiscale, en s'assurant que les profits soient taxés à l'endroit même où ceux-ci sont générés et où a lieu la création de valeur. Une fois ces instruments intégrés au système fiscal français, les gouvernants ne pourront aisément, par le biais de réformes, créer un nouveau système fiscal duquel ces instruments seraient absents.

\footnotetext{
${ }^{53}$ Les deux modèles de convention fiscale internationale les plus répandus sont les modèles OCDE et ONU.

${ }^{54}$ Voir en ce sens : Organisation de CoOpération et de DÉveloppement Économiques, Concurrence fiscale dommageable - Un problème mondial, Paris, Éditions OCDE, 1998, 88 p.

55 Par exemple, la définition du revenu des conventions signées par la France contraint le pays, même si celle-ci va à l'encontre de sa définition interne. Cette dichotomie reprend le débat entre la théorie de la source et la théorie de l'enrichissement. La notion de revenu en droit fiscal français est à mi-chemin entre la conception civiliste qui reprend la théorie de la source, et la conception économique qui reprend celle de l'enrichissement. Cependant en cas d'extranéité, l'application fiscale est dépendante de l'option, figurant dans le modèle OCDE, d'attribuer le droit d'imposer à l'État de la source ou à l'État de la résidence. Si la répartition est historiquement favorable à l'État de résidence, un rééquilibrage pourrait intervenir d'ici 2020 passant par davantage d'imposition au profit de l'État de la source.

56 Voir en ce sens : H. ASCENSIO et C. FOLSCHÉ, «L'OCDE et la coopération fiscale internationale », in SOCIÉTÉ

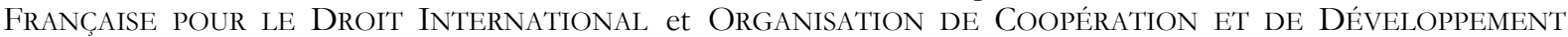
ÉCONOMIQUES (dir.), Le pouvoir normatif de l'OCDE, Journée d'étude de Paris, Paris, A. Pedone, pp. 79-96; ORGANISATION DE COOPÉRATION ET DE DÉVELOPPEMENT ÉCONOMIQUES, Lutter contre l'érosion de la base d'imposition et le transfert de bénéfices, Paris, Éditions OCDE, 2013, 95 p. ; et J. DEHAUSSY et H. AsCENSIO, « Actes unilatéraux et action normative des organisations internationales », JurisClasseur Droit international, 2005, p. 15 et s.
} 
La représentation systématisée du droit étant interne au droit, l'application de la méthode systémique à la fiscalité a permis d'identifier le système fiscal français, composé d'éléments réunis en trois groupes (les impositions de l'enrichissement, celles du patrimoine, et celles de la dépense) entrant en interaction et formant ainsi le triangle, socle structurel du système fiscal français. Toutefois si les aspirations de certains citoyens et politiques convergent de plus en plus vers une remise à plat de toute la fiscalité française pour repartir sur un système plus efficient, elles semblent contrariées par le comportement du système intrinsèquement inerte et résilient. Effectivement, une telle perspective est impossible, car une part du système ne peut être transformée. Il en est ainsi de la structure du système fiscal qui, calqué sur le cycle économique, ne saurait voir sa dynamique interne modifiée. Il en est également de même pour toute la fiscalité confiée à l'Union européenne, pour laquelle la France n'est plus en capacité de changer ses règles internes, contraignant alors une conservation de certains éléments du système. De plus, toutes les modifications ne sont pas envisageables puisqu'il faut respecter les règles européennes, ou plutôt l'encadrement, les interdictions européennes. Les standards internationaux en matière fiscale viennent enfin participer à la résilience du système fiscal français et accompagner une convergence de tous les systèmes fiscaux des pays occidentaux.

La modélisation systémique de la fiscalité a permis d'anticiper l'avenir par l'étude de la dynamique du système fiscal français au gré des réformes. « Tout semble se passer comme si les responsables politiques n'avaient aucune prise réelle sur notre système fiscal $\aleph^{57}$. Il semble alors que tout souhait d'une métamorphose totale de la fiscalité est inenvisageable aujourd'hui. Si l'espérance est, comme le prétendait ARISTOTE : «le songe d'un homme éveillé $~_{58}$, espérer un nouveau système fiscal - espérance au cœur du débat national contemporain - est un songe : l'espérance d'un système fiscal totalement nouveau est vaine.

57 Th. LAMBERT, "La politique fiscale sous la cinquième République: entre réformes et changements », in Th. LAMBERT et alii., Les chantiers fiscaux à engager, Paris, L'Harmattan, Finances publiques, 2002, p. 23.

${ }^{58}$ J. Chevalier, Histoire de la pensée : D'Aristote à Plotin, Vol. 2, Paris, Flammarion, Éditions universitaires, 1991, p. 14. 